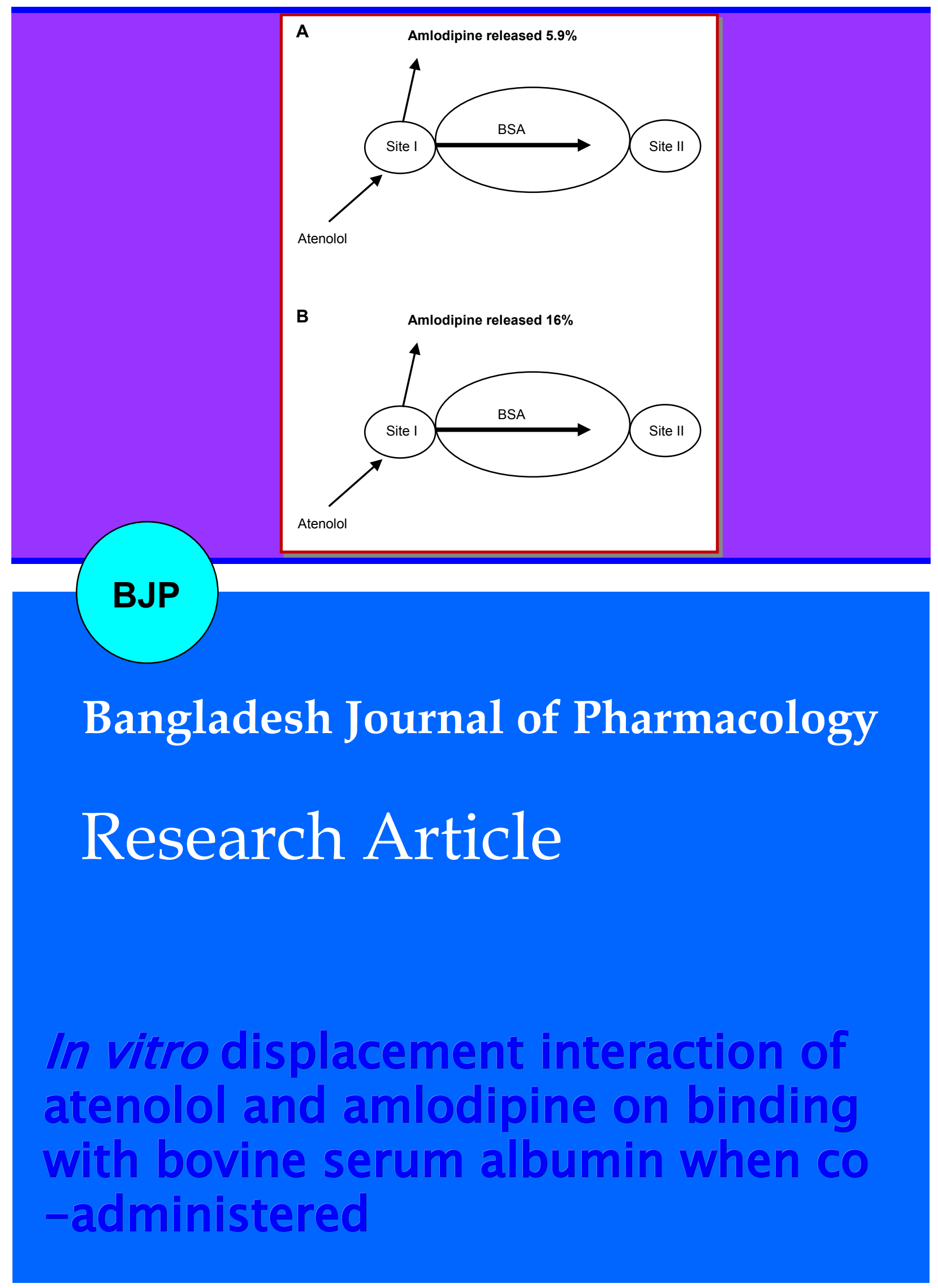


Abstracted/indexed in Academic Search Complete, Asia Journals Online, Bangladesh Journals Online, Biological Abstracts, BIOSIS Previews, CAB Abstracts, Current Abstracts, Directory of Open Access Journals, EMBASE/Excerpta Medica, Google Scholar, HINARI (WHO), International Pharmaceutical Abstracts, Open J-gate, Science Citation Index Expanded, SCOPUS and Social Sciences Citation Index;

ISSN: $1991-0088$

\title{
In vitro displacement interaction of atenolol and amlodipine on binding with bovine serum albumin when co-administered
}

\author{
Md. Ashraful Alam¹, Md. Abdul Awal'1, Mahbub Mostofa', Md. Kamrul Islam² and \\ Nusrat Subhan 3
}

${ }^{1}$ Department of Pharmacology, Bangladesh Agricultural University, Mymensingh 2202, Bangladesh; ${ }^{2}$ Department of Physiology, Bangladesh Agricultural University, Mymensingh 2202, Bangladesh; ${ }^{3}$ Pharmacy Discipline, Khulna University, Khulna 9208, Bangladesh.

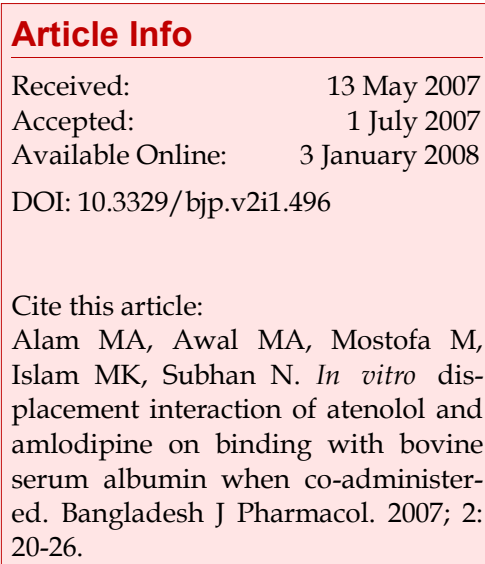

\begin{abstract}
The binding of atenolol (selective $\beta_{1}$-blocker) and amlodipine (calcium channel blocker) to bovine serum albumin (BSA) was studied by equilibrium dialysis method in order to have an insight into the binding chemistry of these two to BSA. Free atenolol concentration was increased due to addition of amlodipine which reduced the binding of the compounds to BSA. However, the free fraction was increased to a level as it was expected from direct competitive displacement while the free atenolol concentration was increased according to increasing the amlodipine concentration when only the BSA was present. The result obtained when the binding site was blocked by sufficient amount of amlodipine was that the increment of free concentration of atenolol was prominent. When no amlodipine was added the free concentration of atenolol was only $28 \%$ whereas this release was $93 \%$ to $98.0 \%$ when amlodipine was added with increasing concentration.
\end{abstract}

\section{Introduction}

It has been revealed by many epidemiological studies including the Framingham study that diabetes mellitus and hypertension are respectively risk factors of cardiovascular diseases and that the coexistence of diabetes mellitus with hypertension considerably increases the risk of cardiovascular diseases. (Epstein and Sowers, 1992; Grossman and Messerli, 1996; American Diabetes Association, 2002) The result of the U.K. Prospective Diabetes Study (UKPDS) suggests that blood pressure control, rather than blood glucose control, is efficient for prevention of macrovascular complications of those of diabetes mellitus, which include stroke and myocardial infarction (UK Prospective Diabetes Study
Group, 1998). The results of the Hypertension Optimal Treatment (HOT)-study on the correlation between optimum target blood pressure levels and the occurrence of cardiovascular events also suggest that it is useful for hypertensive patients with diabetes mellitus to set the target levels lower than those for general hypertension patients (Hansson et al., 1998).

Aggressive antihypertensive therapy needs to be carried out. $\beta$-adrenoceptor blocking drugs are widely used for the treatment of cardiovascular diseases such as arterial hypertension, coronary heart disease and supraventricular and ventricular tachyarrhythmias. They may also be beneficial in the hyperkinetic heart syndrome, hypotensive circulatory disorders, portal hyper- 
tension, hyperthyroiddism, tremor, migraine, anxiety, psychosomatic disorders or glaucoma.

In recent years even patients with heart failure have been successfully treated with $\beta$-blockers initially given at very low doses. Cardiac adverse events are a major cause of morbidity and mortality in patients undergoing non-cardiac surgery (Mangano et al., 1990). The risk is highest among patients with a history of myocardial infarction, angina pectoris, congestive heart failure, arterial hypertension or diabetes mellitus, particularly in the elderly and in patients with unstable symptoms, and the effects of these risk factors appear to be additive. Ischemia during treadmill testing was more effectively suppressed by amlodipine, whereas ischemia during ambulatory monitoring was more effectively suppressed by atenolol. The combination was more effective than either single drug in both settings (Davies et al., 1995).

The current study has been carried out for the initial understanding of the in vitro displacement interaction of the two drug atenolol and amlodipine on binding site of bovine serum albumin. In this study bovine serum albumin (BSA) was used in lieu of human serum albumin because of its low cost and easy availability.

\section{Materials and Methods}

\section{Drug and reagents}

Atenolol, amlodipine besylate (General Pharmaceutical Ltd., Bangladesh). disodium hydrogen phosphate $\left(\mathrm{Na}_{2} \mathrm{HPO}_{4}\right)$, potassium dihydrogen phosphate $\left(\mathrm{KH}_{2} \mathrm{PO}_{4}\right)$, cellulose nitrate membrane (Medicell Internation Ltd. Liverpool Road, London; mol. Wt. 1200 Daltons), bovine serum albumin (BSA) (fatty acid free, fraction V, Mol. Wt. 66,500 from Sigma Chemical Ltd.), warfarin sodium (ACI Pharmaceuticals Ltd.).

\section{Instrument used}

pH Meter (HANNA microprocessor $\mathrm{pH}$ meter, Portugal), SP8-400 UV/VIS spectrophotometer (Thermospectronic, England), metabolic shaking incubator (Clifton shaking bath, Nical Electro Ltd., England.) and micro syringe (well. Liang. Jin. Yang.q.I., China.)

\section{Method used}

Equilibrium Dialysis method was employed in the study (Singlas, 1987).

\section{Site-specific probes method}

We may use different site-specific probes to enhance our understanding of the drug-BSA interaction and thereby characterization of binding sites of the drugs used in the study on the BSA molecule.

Two site-specific probes

Warfarin sodium (site I specific probe) and diazepam (site II specific probe)

Effect of atenolol on amlodipine binding to BSA in presence of site I specific probe warfarin sodium

Two $\mathrm{mL}$ of previously prepared $20 \mu \mathrm{M}$ BSA solution was taken in each of seven cleaned and dried teat tubes. $12 \mathrm{~mL}$ of $10 \mu \mathrm{M}$ warfarin solution was taken in each of seven cleaned and dried test tubes. So that final ratio between protein and warfarin was 1:2 (20:40 $\mu \mathrm{M})$ in each of seven test tubes. Then the site I is sufficiently block by warfarin sodium. The seventh test tube containing only BSA solution was marked as blank. After that amlodipine was added in 6 out of 7 test tubes so that protein, warfarin and amlodipine was 1:2:1 (20:40:20 $\mu \mathrm{M})$. Atenolol was added with an increasing concentration in to five out of six test tubes containing 1:2:1 mixture of protein-warfarin-amlodipine to make the final ratio of protein, warfarin-amlodipine and atenolol 1:2:1:0, 1:2:1:1, 1:2:1:2, 1:2:1:4, 1:2:1:6, 1:2:1:8, $1: 2: 1: 10$. Atenolol was not added to one test tube. The solution mixture were then properly mixed and allowed to stand for $15 \mathrm{~min}$ for the confirmation of maximum binding to BSA. After that the solution was pipetted out and poured in to seven different semi permeable membrane tubes. Both the end of the membrane tubes were clipped and was ensured that there were no leakages. The tubes containing drug mixture were immerged in seven $50 \mathrm{~mL}$ conical flasks containing $30 \mathrm{~mL}$ of phosphate buffer solution of $\mathrm{pH}$ 7.4. The conical flasks were shaked in a shaker at $27^{\circ} \mathrm{C}$ and $20 \mathrm{rpm}$ for about 6 hours uninterruptedly. After shaking samples from each flask were collected. Free concentrations of amlodipine were measured by a UV spectrophotometer.

\section{Effect of atenolol on amlodipine binding to BSA in absence of site I specific probe warfarin sodium}

To perform the experiment the previously described procedure has been followed successively in absence of warfarin sodium. Atenolol was added with an increasing concentration in to five out of six test tubes 
containing 1:1 mixture of protein-amlodipine to make the final ratio of protein, amlodipine and atenolol 1:1:0, 1:1:1, 1:1:2, 1:1:4, 1:1:6, 1:1:8, 1:1:10. Atenolol was not added to one test tube. That is atenolol was not present into the first test tube which contained only proteinamlodipine mixture (1:1). At the end of dialysis, samples were collected from each flask. The free concentrations of amlodipine were measured by a UV spectrophotometer at a wavelength of $235 \mathrm{~nm}$.

Effect of amlodipine on atenolol binding to BSA in presence of site I specific probe warfarin sodium

Two mL of previously prepared $20 \mu \mathrm{M}$ BSA solution was taken in each of seven cleaned and dried teat tubes. $12 \mathrm{~mL}$ of $10 \mu \mathrm{M}$ warfarin solution was taken in each of seven cleaned and dried test tubes. So that final ratio between protein and warfarin was 1:2 $(20: 40 \mu \mathrm{M})$ in each of seven test tubes. Then the site I is sufficiently block by warfarin sodium. The seventh test tube containing only BSA solution was marked as blank. After that atenolol was added in 6 out of 7 test tubes so that protein, warfarin and atenolol was 1:2:1 (20:40:20 $\mu \mathrm{M})$. Amlodipine was added with an increasing concentration in to five out of six test tubes containing 1:2:1 mixture of protein-warfarin-amlodipine to make the final ratio of protein, warfarin-atenolol and amlodipine 1:2:1:0, 1:2:1:1, 1:2:1:2, 1:2:1:4, 1:2:1:6, 1:2:1:8, 1:2:1:10. Amlodipine was not added to one test tube. The solution mixture were then properly mixed and allowed to stand for $15 \mathrm{~min}$ for the confirmation of maximum binding to BSA. After that the solution was pipetted out and poured in to seven different semi permeable membrane tubes. Both the end of the membrane tubes were clipped and was ensured there were no leakages. The tubes containing drug mixture were immerged in seven $50 \mathrm{~mL}$ conical flasks containing $30 \mathrm{~mL}$ of phosphate buffer solution of $\mathrm{pH}$ 7.4. The conical flasks shake in a shaker at $27^{\circ} \mathrm{C}$ and 20 rpm for about 6 hours uninterruptedly. After shaking samples from each flask were collected. Free concentrations of atenolol were measured by a UV spectrophotometer.

Effect of amlodipine on atenolol binding to BSA in absence of site I specific probe warfarin sodium

To perform the experiment the previously described procedure has been followed successively in absence of warfarin sodium. Amlodipine was added with an increasing concentration in to five out of six test tubes containing 1:1 mixture of protein-amlodipine to make the final ratio of protein, atenolol and amlodipine 1:1:0, 1:1:1, 1:1:2, 1:1:4, 1:1:6, 1:1:8, 1:1:10. Atenolol was not added to one test tube. That is amlodipine was not present into the first test tube which contained only protein-atenolol mixture (1:1). At the end of dialysis, samples were collected from each flask. The free concentrations of atenolol were measured by a UV spectrophotometer at a wavelength of $275 \mathrm{~nm}$.

\section{Results}

The drug-drug interaction study of atenolol and amlodipine bound to BSA was conducted in the presence and absence of site specific probe at $27^{\circ} \mathrm{C}$ and $\mathrm{pH}$ 7.4. Atenolol and amlodipine bind considerably with the high affinity association constant but low capacity to site I and with low value of association constant but high capacity to site II on BSA.

In the study of atenolol and amlodipine interaction, the effect of atenolol on amlodipine bound to BSA was determined by in presence and absence of site I specific probe warfarin. Atenolol and amlodipine is site I specific drug so we had used warfarin as a site one specific probe. The effect of atenolol on amlodipine in absence and in presence of site I specific probe warfarin sodium is shown in Figure 1. Free concentration of amlodipine bound to BSA $(1: 2 ; 20: 40 \mu \mathrm{M})$ released upon the addition of atenolol in absence of probe increase from 5.9 to $16.1 \%$. Again in presence of site I specific probe (warfarin) with the increase of atenolol concentration from 0 to $100 \mu \mathrm{M}$ concentration of amlodipine increased from 7.6 to $18.6 \%$ (Figure 1 ).

Again, the effect of amlodipine on atenolol in absence and in presence of site I specific probe warfarin sodium is shown in Figure 2. Free concentration of atenolol bound to BSA $(1: 2 ; 20: 40 \mu \mathrm{M})$ released upon the addition of amlodipine in absence of probe increase from 28 to $93 \%$. Again in presence of site I specific probe (warfarin) with the increase of amlodipine concentration from 0 to $100 \mu \mathrm{M}$ (Figure 2) concentration of atenolol increased from 23.4 to $98.1 \%$.

On the basis of above data obtained during concurrent administration of atenolol and amlodipine added in absence or presence of site I specific probe, warfarin sodium, different models of interactions have been proposed (Figure 3 and 4). 


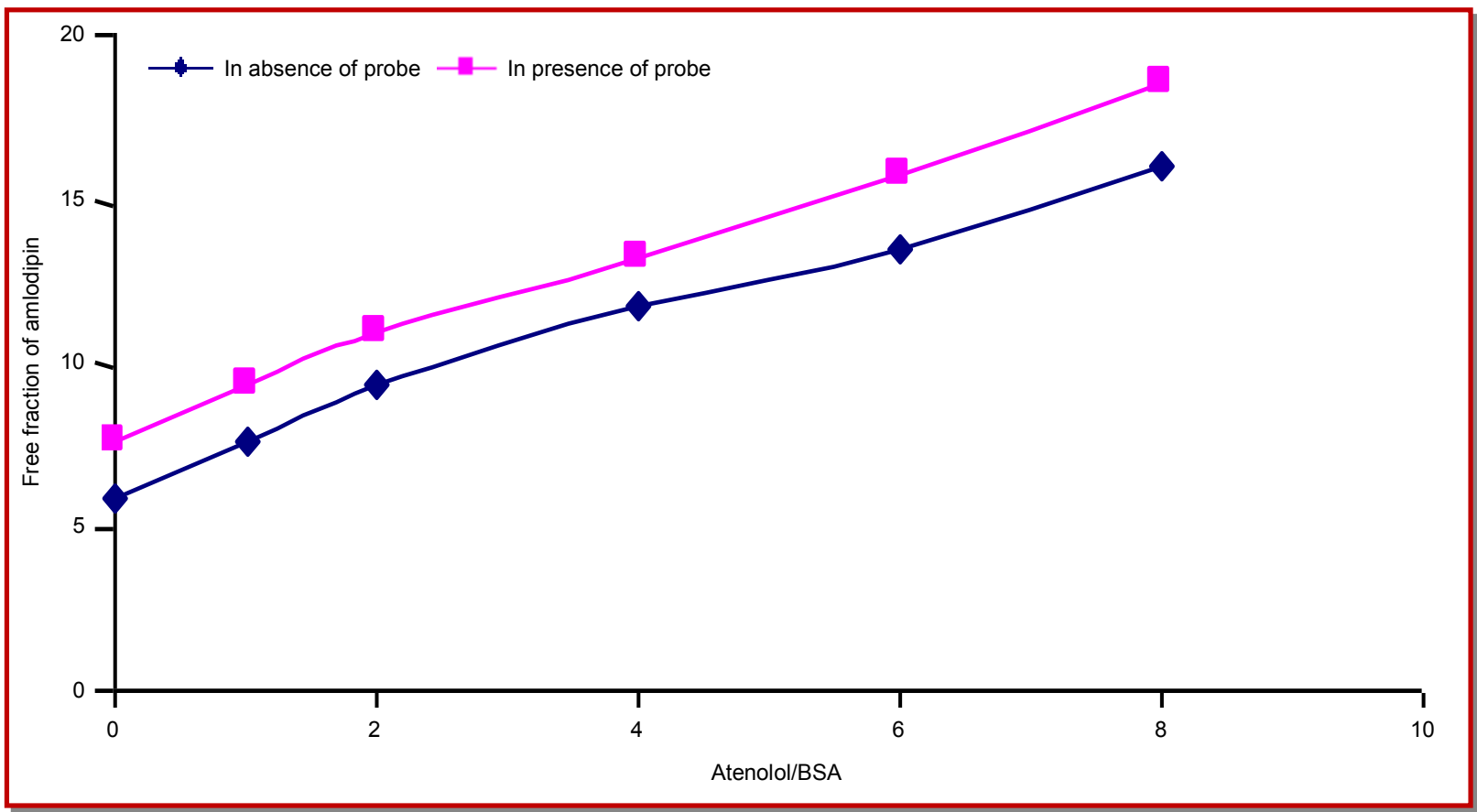

Figure 1: Displacement of amlodipine $(20 \mu \mathrm{M})$ from its binding site to BSA upon addition of increasing amount of atenolol from 0 to $100 \mu \mathrm{M}$ concentration

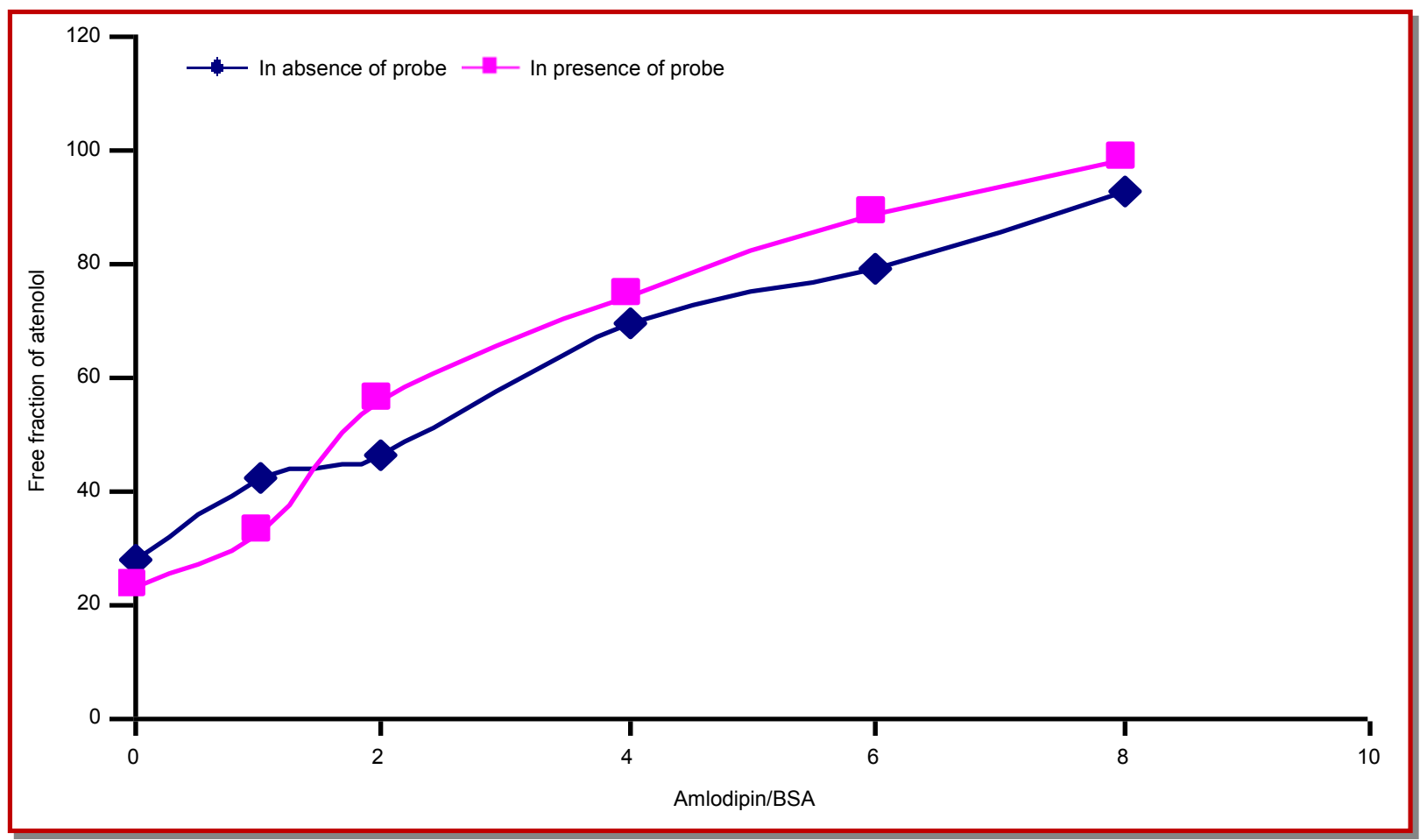

Figure 2: Displacement of atenolol $(20 \mu \mathrm{M})$ from its binding site to BSA upon addition of increasing amount of amlodipine from 0 to $100 \mu \mathrm{M}$ concentration

\section{Discussion}

Plasma protein binding properties are considered as the primary determinants of the pharmacokinetics proper- ties of drugs. Any physiological condition that causes alteration in the albumin binding of the drugs might lead to change in the pharmacokinetics and pharmacological properties of the drugs. Drug-drug interactions 


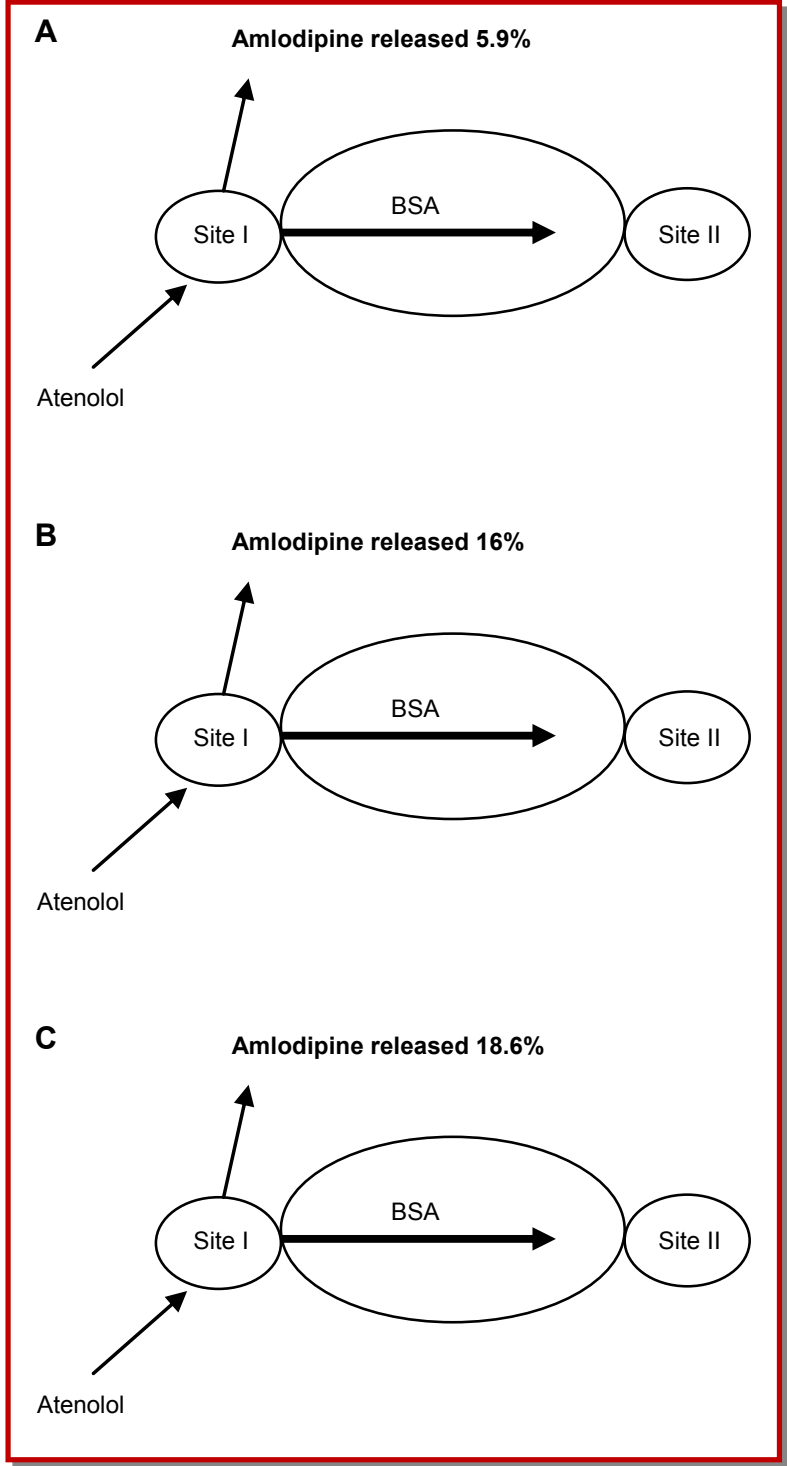

Figure 3: Proposed models of the amlodipine-BSA-atenolol interaction in presence and absence of warfarin (site I specific probe)

Concentrations were used: $[\mathrm{BSA}]=[$ Amlodipine $]=20 \mu \mathrm{M}$; [Atenolol $]$ $=160 \mu \mathrm{M}$; [Warfarin] $=40 \mu \mathrm{M}$. (A) Normal condition of binding; (B) Drug interaction in absence of probe; (C) Drug interaction in presence of probe

thus play a vital role in the extent of plasma-protein binding and consequently the therapeutic effect of the drugs. To evaluate the probable interaction between drugs, the binding sites of the drug on the protein have to be known (Hober and Sjoholm, 1980). Ca antagonists exert the anti-hypertensive action to wide-ranging patients, and are commonly used as antihypertensive drugs through the mechanism of inhibiting calcium entry, which triggers constriction in vascular smooth muscle cells. Ca antagonists are recommended as a

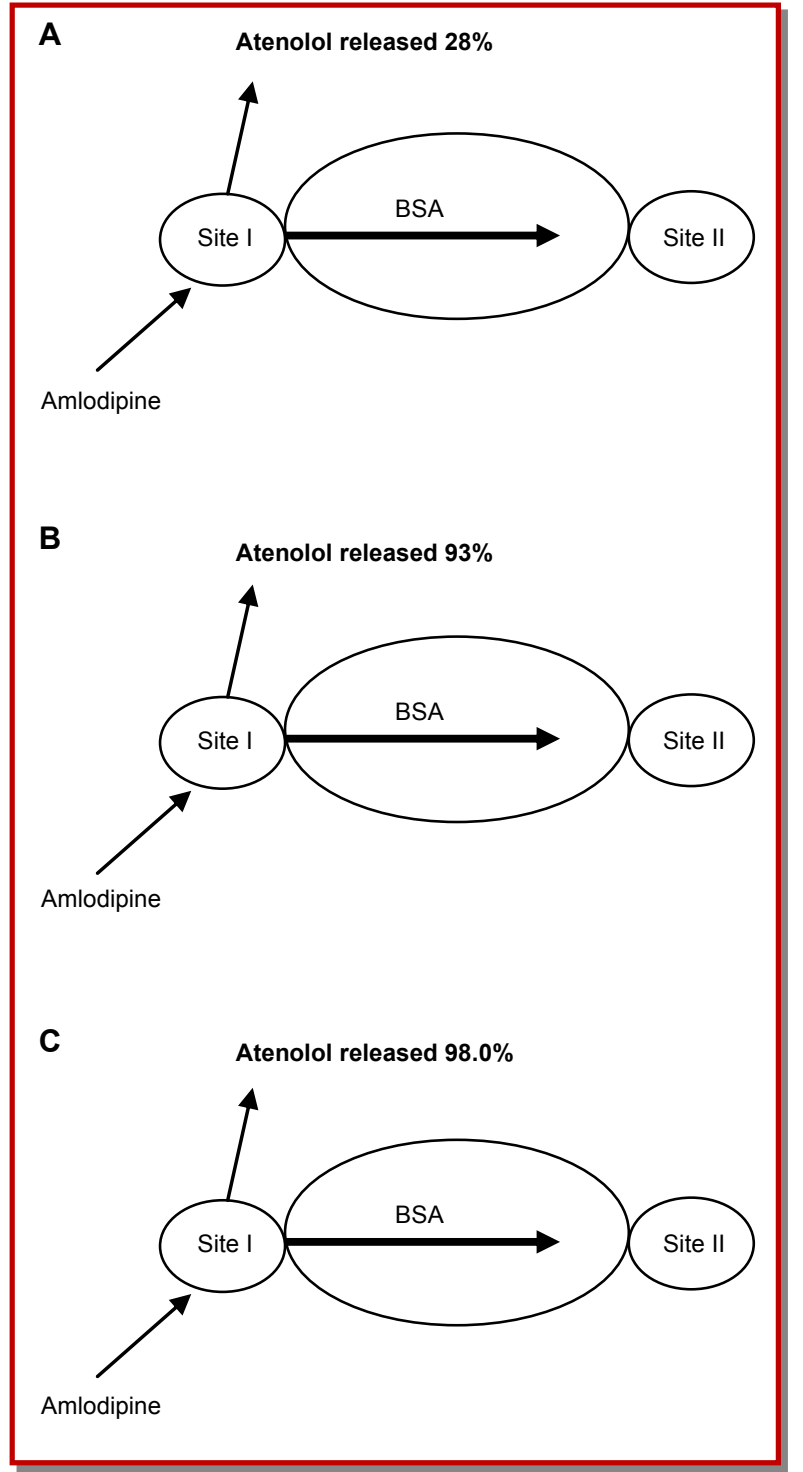

Figure 4: Proposed models of the amlodipine-BSA-atenolol interaction in presence and absence of warfarin (site I specific probe)

Concentrations were used: $[\mathrm{BSA}]=[$ Atenolol $]=20 \mu \mathrm{M} ;$ [Amlodipine $]$ $=160 \mu \mathrm{M}$; $[$ Warfarin $]=40 \mu \mathrm{M}$. (A) Normal condition of binding; (B) Drug interaction in absence of probe; (C) Drug interaction in presence of probe

therapeutic medicine for hypertensive patients with diabetes mellitus, because they have no adverse influence on lipid metabolism or glucose metabolism (Tumoliehto et al., 1999; Epstein et al., 1992; Tatti et al., 1998; Alderman et al., 1998; Pahor et al., 1998a; Pahor et al., 1998b). Several studies was designed to investigate the possible synergism of atenolol and amlodipine on lowering and stabilizing blood pressure in spontaneously hypertensive rats demonstrated that there is a synergistic effect between atenolol and 
amlodipine in lowering and stabilizing the blood pressure. The synergistic effect is highest when the dose proportion of the two drugs is 10:1 (Xu et al., 2004). Moreover, amlodipine decreased TNF- $\alpha$, increased IL-6 and did not affect IL-1 $\beta$ level. Atenolol did not influence TNF- $\alpha$ and IL-1 $\beta$, but raised IL-6 in SHR. Additionally, amlodipine decreased total cholesterol level without changing HDL cholesterol level whereas atenolol did not influence lipid levels (Andrzejczak et al., 2006). Amlodipine is effective in patients with angina inadequately controlled by atenolol alone, and does not interfere with cardiac rhythm or function (Woodmansey et al., 1993). Drugs are bound to plasma protein at sites located on the surface of the protein. The idea of binding sites is suggested by the relative size of the drugs and proteins. Protein binding of a drug is governed by the complex of the drug, which form with the protein. There are two main type of protein binding i.e. strong affinity binding to a small number of sites and weak affinity binding to a large number of sites. Since binding is almost exclusively to albumin and the number of sites available is limited, the protein binding of some drugs depends on the plasma albumin concentration.

\section{Conclusion}

The displacement of atenolol and amlodipine from its protein binding site due to the concurrent administration of these two drugs in in vitro condition significantly increase the free drug concentration. The increment is higher for atenolol than amlodipine. This phenomenon will be a potential mechanism for drug synergism as atenolol-amlodipine combined dosage form that has been established for the treatment or can be recommended as a therapeutic medicine for hypertensive patients with diabetes mellitus, because they have little adverse influence on lipid metabolism or glucose metabolism.

\section{References}

Alderman M, Madhavan S, Cohen H. Calcium antagonists and cardiovascular events in patients with hypertension and diabetes. Lancet 1998; 351: 216-17.

American Diabetes Association. Treatment of hypertension in adults with diabetes. Diabetes Care, 2002; 25(Suppl 1): S7173.
Andrzejczak DG, Rska D, Czarnecka E. Influence of amlodipine and atenolol on lipopolysaccharide (LPS)induced serum concentrations of TNF-alpha, IL-1beta, IL-6 in spontaneously hypertensive rats (SHR). Pharmacol Rep. 2006; 58: 711-19.

Davies RF, Habibi H, Klinke WP, Dessain P, Nadeau C, Phaneuf DC, Lepage S, Raman S, Herbert M, Foris K, et al. Effect of amlodipine, atenolol and their combination on myocardial ischemia during treadmill exercise and ambulatory monitoring. Canadian amlodipine/atenolol in silent ischemia study (CASIS) investigators. J Am Coll Cardiol. 1995; 25: 619-25.

Epstein M, Sowers JR. Diabetes mellitus and hypertension. Hypertension 1992; 19: 403-18.

Epstein M. Calcium antagonists and renal protection: Current status and future perspectives. Arch Intern Med. 1992; 152: 1573-84

Grossman E, Messerli FH. Diabetic and hypertensive heart disease. Ann Intern Med. 1996; 125: 304-10.

Hansson L, Zanchetti A, Carruthers SG, Björn Dahlöf, Elmfeldt D, Julius S, Ménqrd J, Rahn KH, Wedel H, Westerling WS. Effects of intensive blood-pressure lowering and aspirin in patients with hypertension: Principal results of the Hypertension Optimal Treatment (HOT) randomized trial. Lancet 1998; 351: 1755-62.

Hober A, Sjoholm I. The binding sites on human serum albumin for some non-steroidal anti-inflammatory drugs. Mol Pharmacol. 1980; 18: 421-26.

Mangano DT, Browner WS, Hollenberg M, London MJ, Tubau JF, Tateo IM. Association of perioperative myocardial ischemia with cardiac morbidity and mortality in men undergoing noncardiac surgery. N Engl J Med. 1990; 323: 1781-88

Pahor M, Kritchevsky SB, Zuccala G, Guralnik JM. Diabetes and risk of adverse events with calcium antagonists. Diabetes Care, 1998a; 21: 193-94.

Pahor M, Psaty BM, Furberg CD. Treatment of hypertensive patients with diabetes. Lancet 1998b; 351: 689-90.

Singlas E. In: Determination of protein binding and its characteristics: Protein binding of drugs, 2nd ed. Switzerland, Hoffmann-La Roche, 1987, pp 25-31.

Tatti P, Pahor M, Byington RB. Outcome results of the Fosinopril versus Amlodipine Cardiovascular Events Randomized Trial (FACET) in patients with hypertension and NIDDM. Diabetes Care, 1998; 21: 597-603.

Tumoliehto J, Rastenyte D, Birkenhanger WH, Thijs L, Antikainen R, Bulpitt CJ, Fletcher AE, Forette F, Goldhaber A, Palatini P, Sarti C, Fagard R. Effect of calcium-channel 
blockade in older patients with diabetes and systolic hypertension. N Engl J Med. 1999; 340: 677-84.

UK Prospective Diabetes Study Group. Efficacy of atenolol and captopril in reducing risk of macro-vascular and microvascular complications in type 2 diabetes. BMJ. 1998; 317: 713 -20 .
Woodmansey PA, Stewart AG, Morice AH, Channer KS. Amlodipine in patients with angina uncontrolled by atenolol. Eur J Clin Pharmacol. 1993; 45: 107-11.

Xu LP, Shen FM, Shu H, Miao CY, Jiang YY, Su DF. Synergism of atenolol and amlodipine on lowering and stabilizing blood pressure in spontaneously hypertensive rats. Fundamental Clin Pharmacol. 2004; 18: 33-38. 